\title{
Profiling of inhibitory immune checkpoints in glioblastoma: Potential pathogenetic players
}

\author{
SALVO DANILO LOMBARDO ${ }^{1}$, ALESSIA BRAMANTI ${ }^{2}$, ROSELLA CIURLEO $^{2}$, \\ MARIA SOFIA BASILE ${ }^{2}$, MANUELA PENNISI $^{3}$, RITA BELLA $^{4}$, KATIA MANGANO ${ }^{3}$, \\ PLACIDO BRAMANTI $^{2}$, FERDINANDO NICOLETTI ${ }^{3}$ and PAOLO FAGONE ${ }^{3}$

\footnotetext{
${ }^{1}$ CeMM Research Center for Molecular Medicine of the Austrian Academy of Sciences, A-1090 Vienna, Austria; ${ }^{2}$ IRCCS Centro Neurolesi Bonino Pulejo, I-98124 Messina; Departments of ${ }^{3}$ Biomedical and Biotechnological Sciences and ${ }^{4}$ Medical Sciences, Surgery and Advanced Technologies, University of Catania, I-95123 Catania, Italy
}

Received July 30, 2020; Accepted October 6, 2020

DOI: $10.3892 / \mathrm{ol} .2020 .12195$

\begin{abstract}
Glioblastoma (GBM) represents the most frequent glial tumor, with almost 3 new cases per 100,000 people per year. Despite treatment, the prognosis for GBM patients remains extremely poor, with a median survival of 14.6 months, and a 5-year survival less than 5\%. It is generally believed that GBM creates a highly immunosuppressive microenvironment, sustained by the expression of immune-regulatory factors, including inhibitory immune checkpoints, on both infiltrating cells and tumor cells. However, the trials assessing the efficacy of current immune checkpoint inhibitors in GBM are still disappointing. In the present study, the expression levels of several inhibitory immune checkpoints in GBM (CD276, VTCN1, CD47, PVR, TNFRSF14, CD200, LGALS9, NECTIN2 and CD48) were characterized in order to evaluate their potential as prognostic and eventually, therapeutic targets. Among the investigated immune checkpoints, TNFRSF14 and NECTIN2 were identified as the most promising targets in GBM. In particular, a higher TNFRSF14 expression was associated with worse overall survival and disease-free survival, and with a lower Th1 response.
\end{abstract}

\section{Introduction}

According to the World Health Organization (WHO) classification of the central nervous system (CNS) tumors, glioblastoma (GBM) is defined as a grade IV astrocytoma (1). GBM represents the most malignant glioma and it is characterized by necrosis, neovascularization and histological heterogeneity (2). GBM represents the most frequent glial tumor, with almost 3 new cases per 100,000 people per year (3). The current stan-

Correspondence to: $\mathrm{Dr}$ Paolo Fagone, Department of Biomedical and Biotechnological Sciences, University of Catania, I-95123 Catania, Italy

E-mail: paolofagone@yahoo.it

Key words: glioblastoma, immune checkpoint, inhibitory checkpoints, astrocytoma, CD276, VTCN1, CD47, PVR, TNFRSF14, CD200, LGALS9, NECTIN2, CD48 dard of care for GBM consists of surgical resection, followed by radiotherapy and chemotherapy with temozolomide (4). Despite treatment, the prognosis for GBM patients remains extremely poor, with a median survival period of 14.6 months, and the 5-year survival is less than 5\% (4).

In recent years, great progress has been made in the area of immunotherapy and accumulating preclinical and clinical data seem to suggest potential novel therapeutic avenues for GBM patients $(5,6)$. It is generally believed that GBM creates a highly immunosuppressive/immuneregulatory microenvironment. Several checkpoint molecules capable of inhibiting the immune responses against neo-antigens, including CTLA4 and PD1/PDL-1, are expressed on both T cells and cancer cells. Immune checkpoint inhibitors, such as nivolumab, ipilimumab and pembrolizumab, have strikingly improved patient survival in solid tumors, such as non-small lung cancer and melanoma. However, the trials assessing the efficacy of immune checkpoint inhibitors in GBM are still disappointing (7). A retrospective study of the use of pembrolizumab in the treatment of recurrent CNS tumors, including GBM, demonstrated that patients treated with Pembrolizumab did not have improved survival (7). Another Phase III randomized trial comparing radiation and concomitant temozolomide with or without nivolumab showed that no progression-free survival benefits were obtained by the addition of nivolumab. However, in a Phase II trial, preoperative administration of nivolumab increased chemokine expression and T-cell receptor clonal diversity, which likely promotes immune-cell infiltration and antitumor immune response (7).

It is reasonable that targeting multiple immune checkpoints in combination with cytotoxic drugs could represent a promising strategy for GBM. The present study characterized the expression levels of several inhibitory immune checkpoints in GBM (i.e., CD276, VTCN1, CD47, PVR, TNFRSF14, CD200, LGALS9, NECTIN2 and CD48) in order to evaluate their prognostic value. Moreover, their potential effects in regulating immune-cell infiltration was investigated.

\section{Materials and methods}

Profiling of inhibitory immune checkpoints in GBM. In order to evaluate the expression levels of inhibitory immune check- 

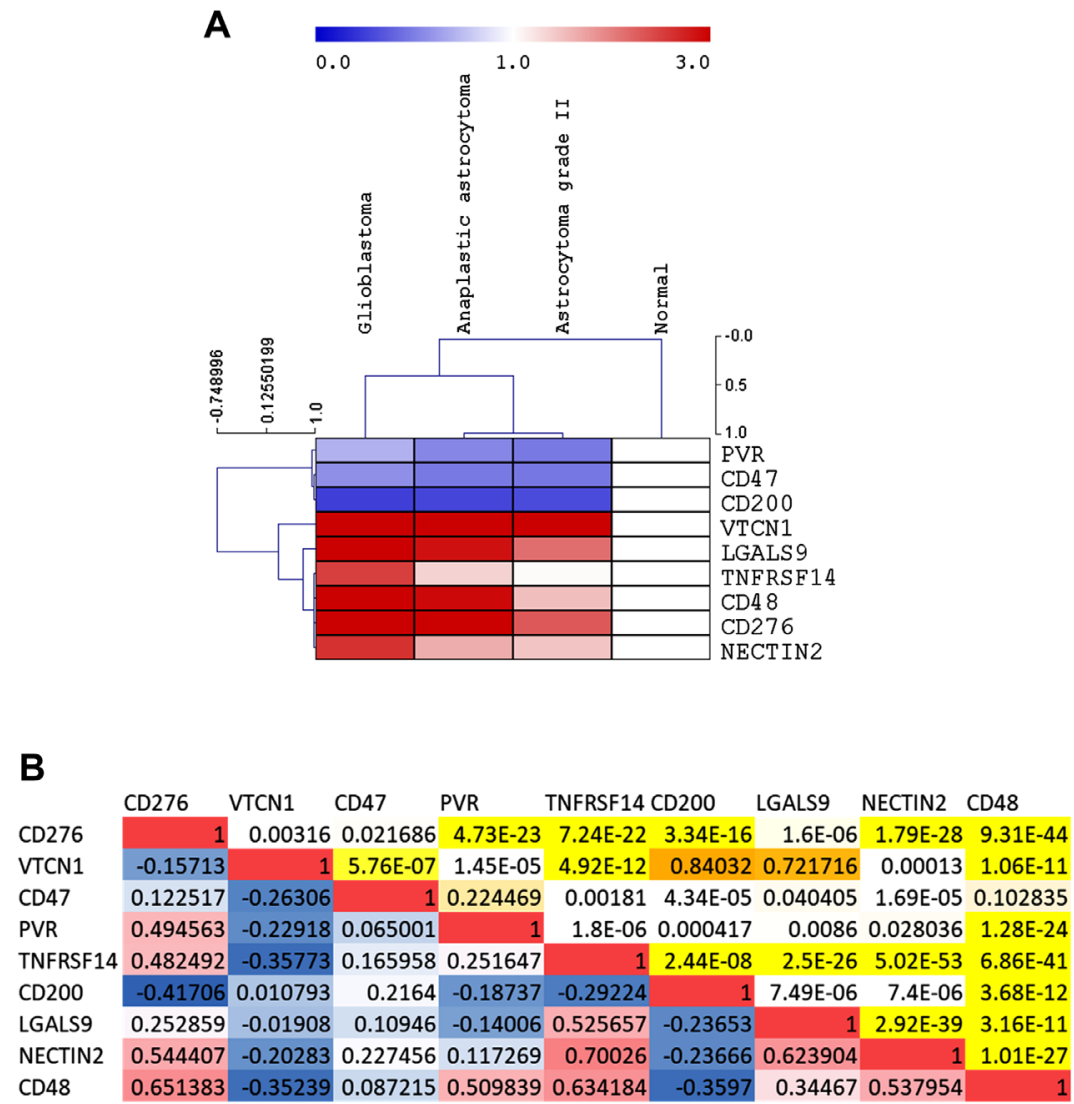

Figure 1. Expression of immune checkpoints in glioblastoma. Relative expression levels of the selected inhibitory immune checkpoints in glioblastoma, lower grade astrocytomas and normal brain samples are presented as heatmap (A). Correlation of the selected inhibitory immune checkpoints (B). Pearson correlation coefficient is presented in blue-red gradient and significance in yellow gradient.

points in GBM as compared to lower grade astrocytomas and normal brain samples, RSEM-normalized RNA Seq data were downloaded from the The Cancer Genome Atlas (TCGA) databank. Selected genes were CD276, VTCN1, CD47, PVR, TNFRSF14, CD200, LGALS9, NECTIN2 and CD48. Complete clinical data of the patients were retrieved and only data from primary tumors, with no neoadjuvant therapy prior to excision, were selected. Data were subjected to logarithmic transformation and Linear Model for Microarray Analysis (LIMMA) was used to assess statistical significance for the differences among cancer types. Overall, this study comprised 153 GBM samples, 130 anaplastic astrocytoma (grade III) samples, 63 astrocytoma (grade II) samples and 5 normal brain samples. The results shown here are based upon data generated by the TCGA Research Network (https://www. cancer.gov/tcga). TCGA Ethics \& Policies were originally published by the National Cancer Institute.

Survival analysis. Samples were stratified in quartiles based on the expression of the genes of interest and samples in the upper and lower quartiles were selected for comparison. Kaplan-Meier curves were constructed for overall survival and disease-free survival and its significance analyzed by log-rank (Mantel-Cox) test.

Computational deconvolution of infiltrating immune cells. In order to evaluate the relative proportions of the infiltrating immune cell subsets in GBM samples diverging for the expression of the selected immune checkpoints and stratified in accordance to survival analysis, we performed a computational deconvolution analysis. The web-based utility, xCell, was used. It is a computational tool that is able, by using gene signatures, to infer the presence in a sample of various cell types, including immature dendritic cells (iDCs), conventional DCs (cDCs), active DCs (aDCs), plasmacytoid DCs (pDCs), B cells, $\mathrm{CD}^{+}{ }^{+}$naive $\mathrm{T}$ cells, memory B cells, plasma cells, Th1 cells, Th2 and Treg cells and macrophages (8).

Statistical analysis. Gene expression differences were evaluated using LIMMA on log-transformed RSEM-normalized expression values. FDR $<0.05$ was considered for statistical significance. Gene expression was visualized as heatmap, using the group mean value. Clustering was performed for both sample groups and genes of interest, using Pearson correlation as distance metrics. Correlation analysis was performed using the Pearson's correlation test. Survival analysis was performed using Kaplan-Meier and its significance analyzed by the log-rank (Mantel-Cox) test. For the analysis, $\mathrm{P}<0.05$ was considered to indicate a statistically significant difference. Statistical analysis was performed with GraphPad Prism 8 (GraphPad Software, Inc.) and SPSS 24 (IBM Corp.).

\section{Results}

Expression of inhibitory immune checkpoints in GBM. A significant upregulation in the expression levels of CD276, 


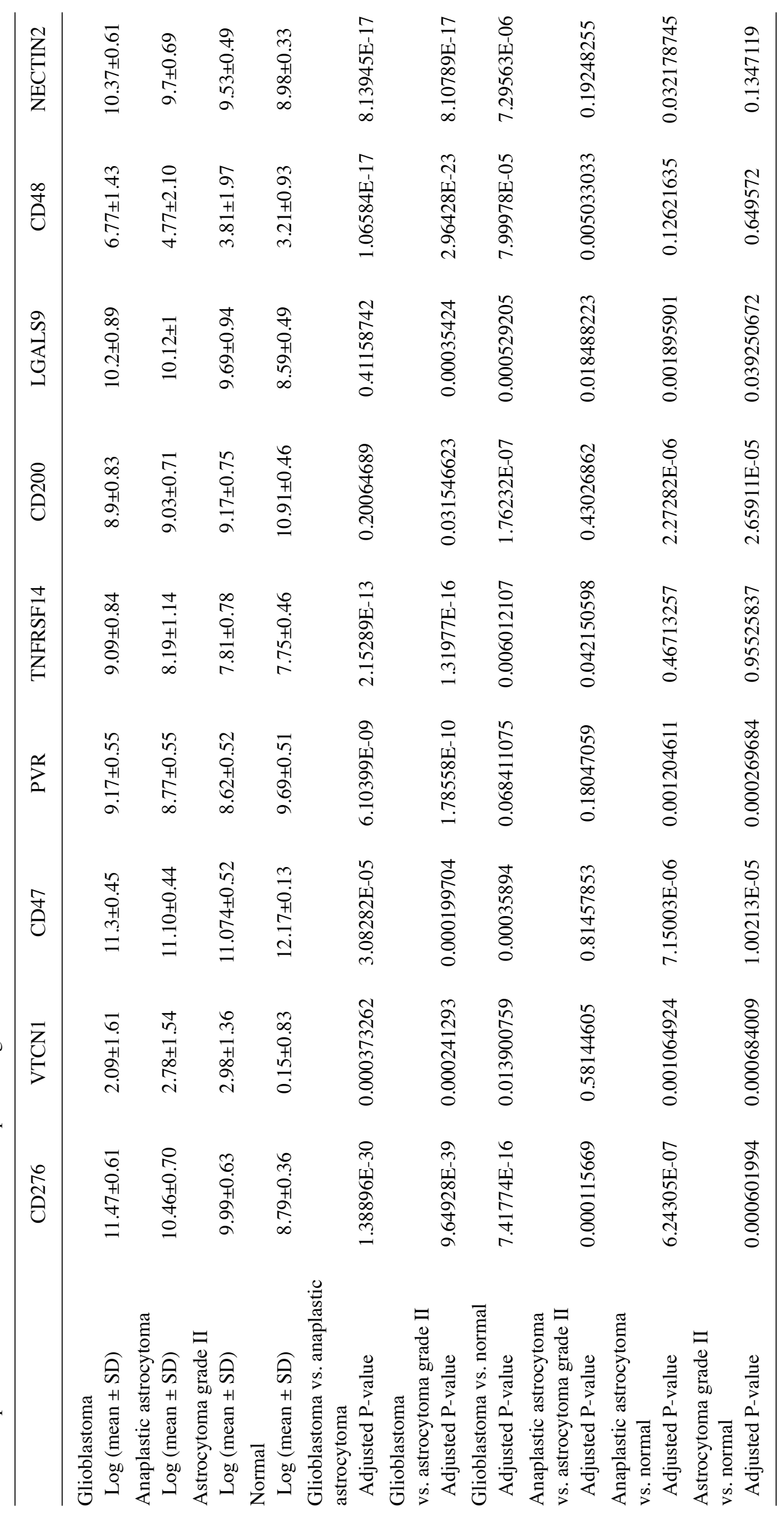




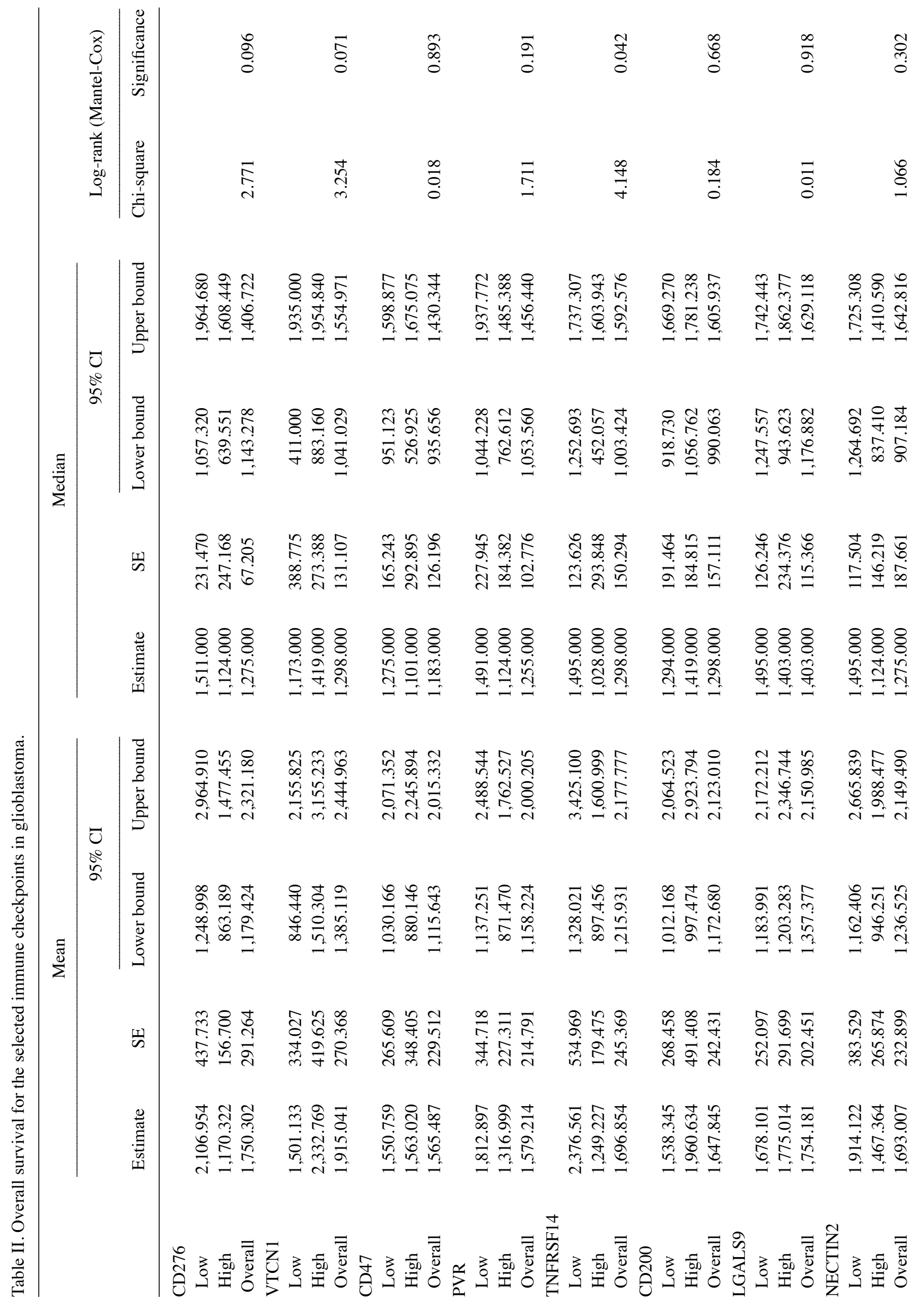




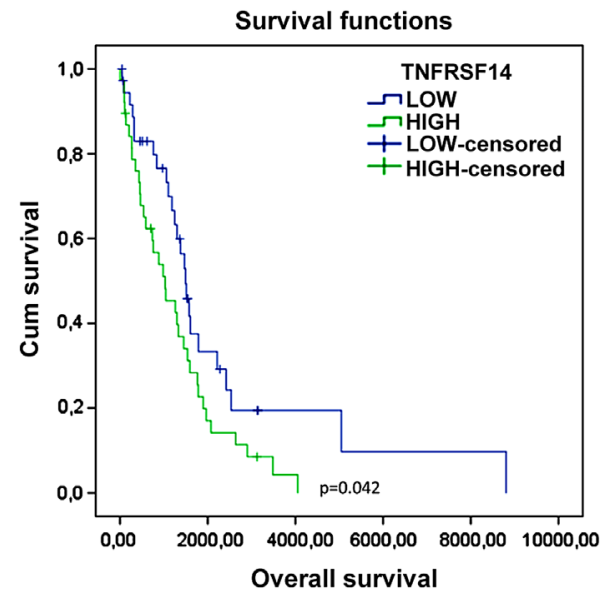

Figure 2. Effect of immune checkpoint expression on overall survival in glioblastoma. Kaplan-Meier curve for the overall survival of glioblastoma patients stratified on the expression levels of TNFRSF14.
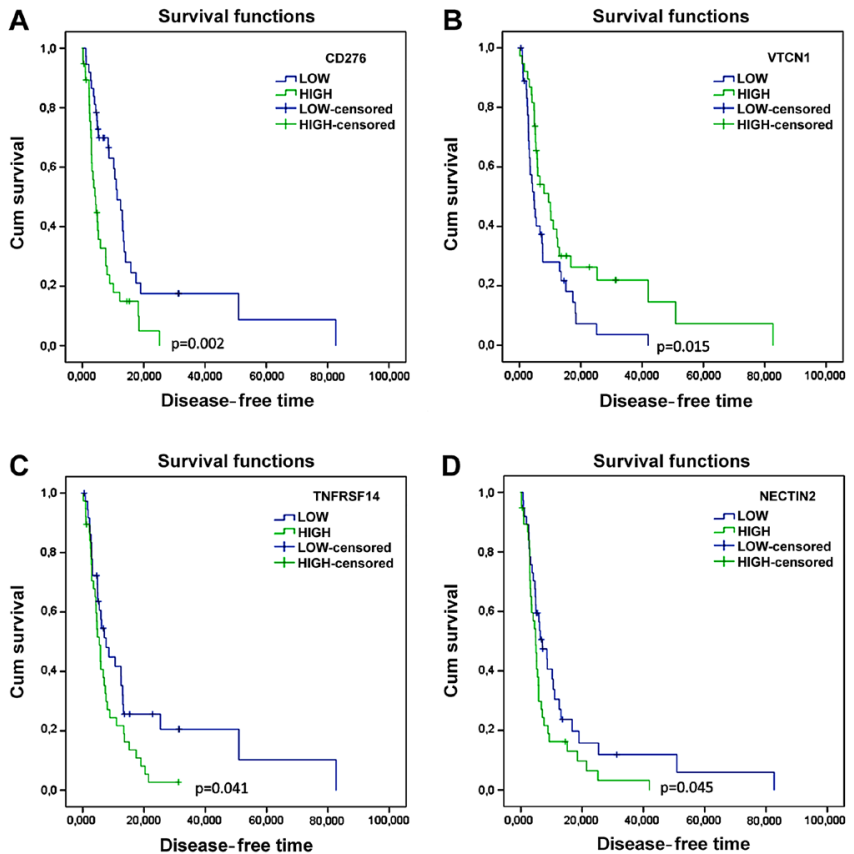

Figure 3. Effect of immune checkpoint expression on disease-free survival in glioblastoma. (A) Kaplan-Meier curve for the disease-free survival of glioblastoma patients stratified on the expression levels of CD276; (B) Kaplan-Meier curve for the disease-free survival of glioblastoma patients stratified on the expression levels of VTCN1; (C) Kaplan-Meier curve for the disease-free survival of glioblastoma patients stratified on the expression levels of TNFRSF14; (D) Kaplan-Meier curve for the disease-free survival of glioblastoma patients stratified on the expression levels of NECTIN2.

VTCN1, TNFRSF14, LGALS9, NECTIN2 and CD48 was observed in GBM as compared to normal brain samples (Fig. 1A, Table I). On the contrary, a significant downregulation of CD47 and CD200 was observed in GBM as compared to normal brain samples, while a trend of downregulation was observed for PVR (Fig. 1A, Table I). Along the same lines, with the exception of LGALS9 and CD200, a significant modulation in the expression levels of the investigated immune checkpoints was observed between the GBM and anaplastic astrocytoma groups of samples (Fig. 1A, Table I). Moreover, 


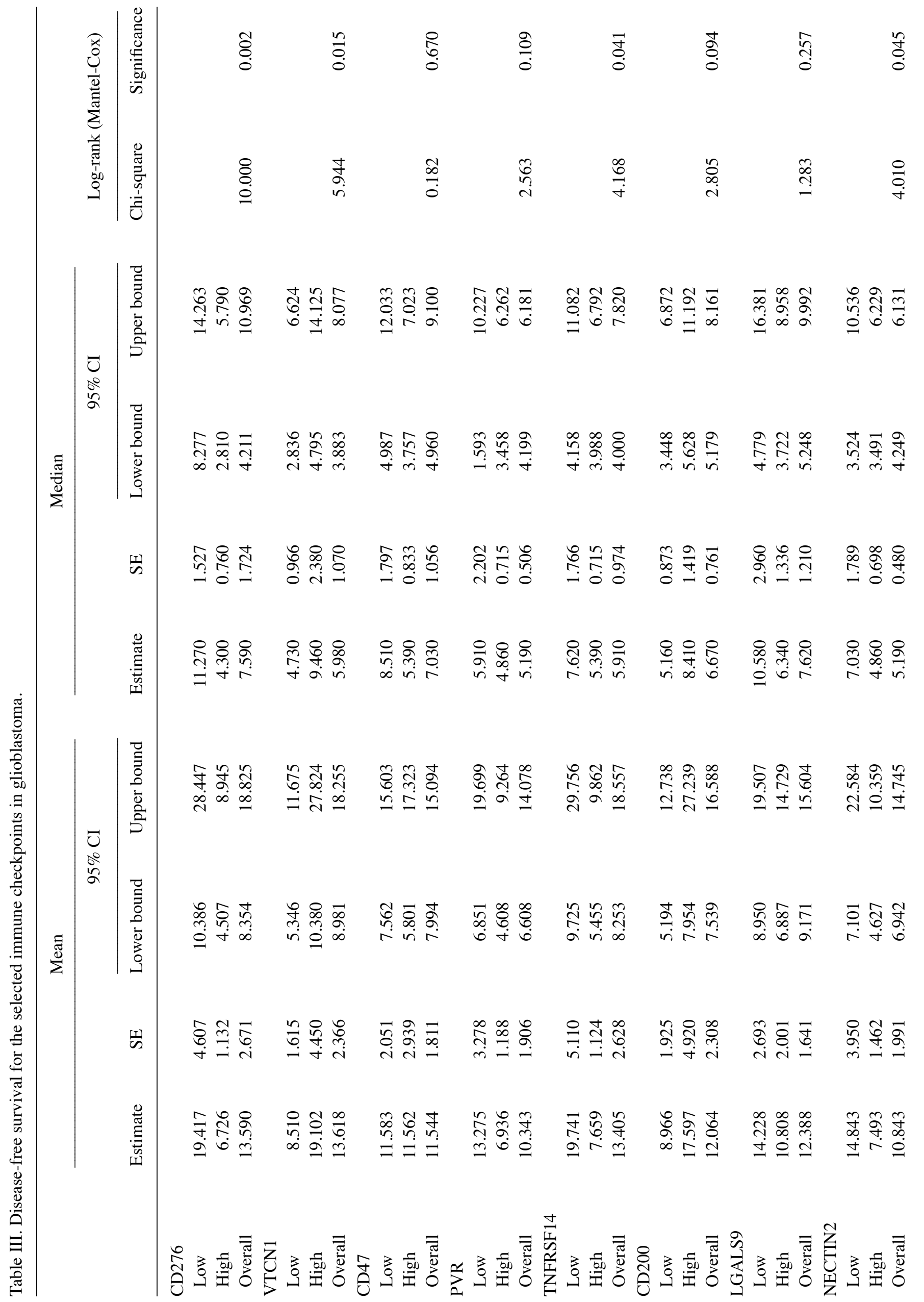


CD276, TNFRSF14, LGALS9 and CD48 resulted significantly upregulated in anaplastic astrocytoma samples as compared to grade II astrocytomas (Fig. 1A, Table I). A significant direct correlation was observed for CD276, PVR, TNFRSF14, NECTIN2 and CD48 (Fig. 1B). Among the GBM samples, a significant negative correlation was instead observed between VTCN1 and PVR, NECTIN2 and CD48 (Fig. 1B).

Survival analysis. Samples were stratified in quartiles based on the expression of the genes of interest, and samples in the upper and lower quartiles were selected for comparison. As shown in Table II and Fig. 2, higher expression levels of TNFRSF14 in GBM were associated to a significantly lower overall survival. No significance was observed for any of the other immune checkpoints. Accordingly, higher TNFRSF14 levels were associated to a shorter disease-free time (Fig. 3 and Table III). Lower levels of CD276 and NECTIN2 were also significantly associated to better disease-free time (Fig. 3 and Table III). Unexpectedly, higher levels of VTCN1 were associated to a longer disease-free time (Fig. 3 and Table III).

Deconvolution analysis. Deconvolution analysis of cell infiltration in GBM was performed on samples dichotomized on the expression levels of the immune checkpoints associated to a significant modulation of survival, i.e., CD276, VTCN1, TNFRSF14 and NECTIN2. As shonw in Fig. 4, higher levels of CD276, TNFRSF14 and NECTIN2 were associated with a significant lower proportion of infiltrating plasma cells. Higher VTCN1 levels were associated to higher proportions of infiltrating plasma cells, along with higher infiltration of Th1, aDCs and cDCs (Fig. 4B). Samples with high expression levels of TNFRSF14 were characterized by a significant lower infiltration of Th1 cells and $\mathrm{CDC}$, and higher proportions of iDCs, aDCs, pDCs and of macrophages (both M1 and M2) (Fig. 4C). A significantly higher infiltration of iDCs, aDCs and M1 macrophages, along with reduced proportions of Th1, Th2 and CD8 T cells, were observed in GBM samples with high NECTIN2 expression levels (Fig. 4D).

\section{Discussion}

Conventional immune checkpoint inhibitors, Nivolumab/ Pembrolizumab for PD-1/PDL1 blockade or Ipilimumab for CTLA4, have proven beneficial effects on the clinical course of different cancer types, including metastatic melanoma, non-small cell lung cancer, renal cell carcinoma, and Hodgkin lymphoma (9-11). However, these treatments have often failed in gliomas (12-14). A possible explanation for this outcome seems to be due to two main glioma features: the low tumor mutational burden (TMB) and a highly immunosuppressive microenvironment. Identifying genomic markers of response to immune checkpoint may benefit cancer patients by providing predictive biomarkers for patient stratification and identifying resistance mechanisms for therapeutic targeting.

The present investigation evaluated the potential role of a series of inhibitory immune checkpoints not previously studied or only marginally characterized in GBM, i.e., CD276, VTCN1, CD47, PVR, TNFRSF14, CD200, LGALS9, NECTIN2 and CD48. To this aim, a computational analysis of RNA-seq data obtained from the TCGA (The Cancer Genome 

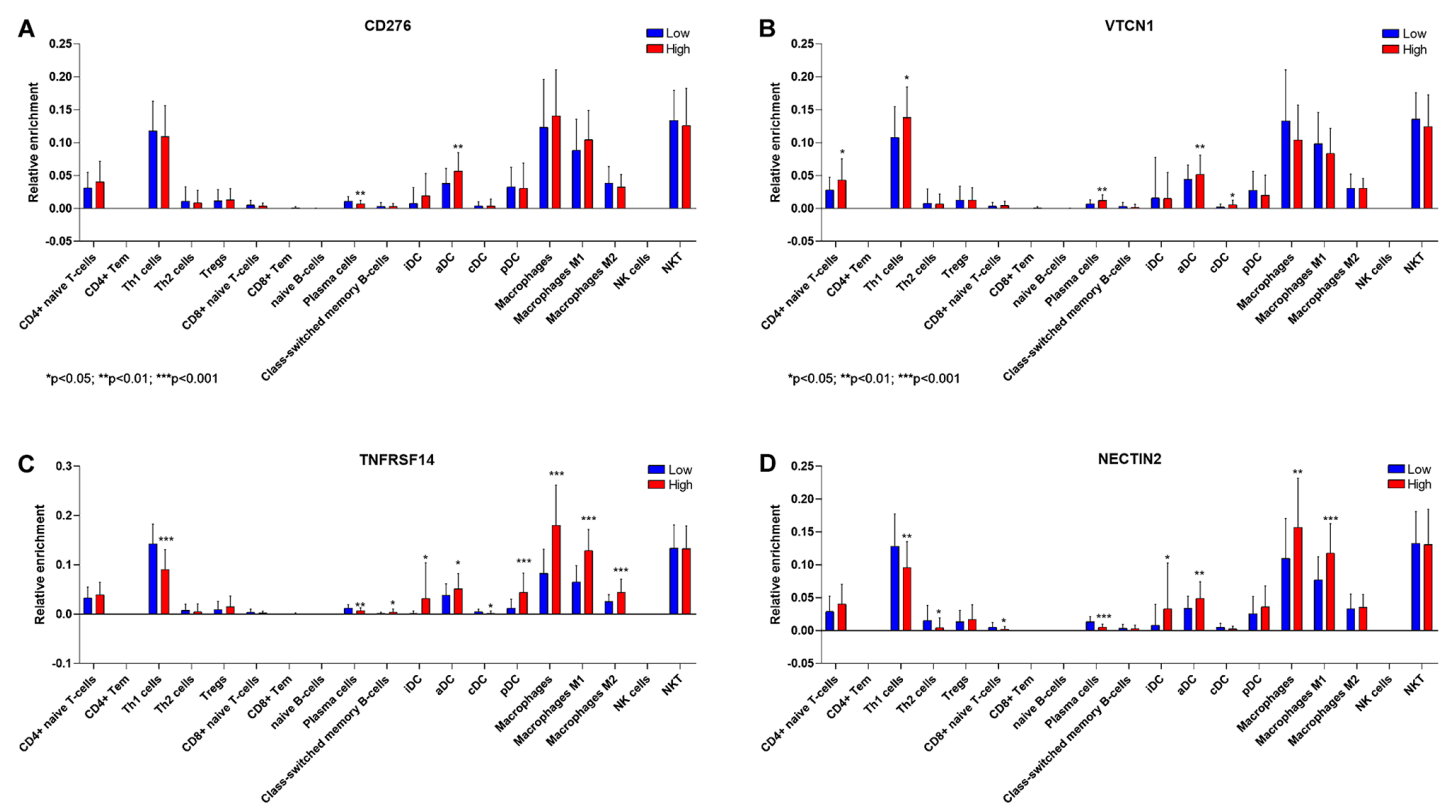

Figure 4. Deconvolution analysis of infiltrating immune cells in glioblastoma. Infiltrating immune cell populations were predicted using the web-based deconvolution analysis utility, xCell, for glioblastoma patients stratified on the expression of (A) CD276, (B) VTCN1, (C) TNFRSF14 and (D) NECTIN2. ${ }^{*} \mathrm{P}<0.05 ;{ }^{* *} \mathrm{P}<0.01 ;{ }^{* * * *} \mathrm{P}<0.001$.

Atlas) database was performed. Whole-genome expression data was largely used (15) to identify pathogenic pathways and therapeutic targets for several disorders, including autoimmune diseases (16-23) and cancer (24-29).

We found that VTCN1 and CD200 are highly over-expressed in GBM, anaplastic astrocytoma and astrocytoma grade II compared to normal brain. Previously, Yao et al (30) showed that VTCN1 has a crucial role in the creation and maintenance of the immunosuppressive microenvironment in gliomas, correlating with prognosis and malignant grades. Furthermore, lower levels of VTCN1 are associated with a higher survival in a clinical trial of DC based vaccination (31). This is in contrast with our observations, which appears to show a protective role for VTCN1 in GBM. The reasons for this counterintuitive data is currently object of further exploration.

On the contrary, CD200 expression levels resulted in significantly reduced astrocytomas in comparison to normal brain. CD200 is a type I transmembrane glycoprotein that plays an inhibitory role in the activation of microglia. For this reason, many studies have shown that its expression is enhanced in brain tumors (32), and especially in higher grade tumors (33). However, its role is still controversial, indeed in the same study Wang et al (33) found that CD200 down-expression can lead to a particular microglia tumor microenvironment that promotes tumor progression, in agreement with our results. Recent studies in dogs also showed that targeting CD200, enhanced the capacity of antigen-presenting cells to prime T-cells to mediate an anti-glioma response (34).

PVR and CD47 were also found down-expressed in astrocytomas when compared to normal brain, while higher levels of expression were found for LGALS9, TNFRSF14, CD48, CD276 and NECTIN2. PVR has been described as regulator of cell adhesion in a rat model of GBM (35) and a recent study in mice proved that the combination of anti-PD-1 and anti-PVR leads to a better survival (36).
CD47 is a member of the immunoglobulin superfamily that activates the signal regulatory protein- $\alpha$ (SIRP- $\alpha$ ) expressed on macrophages, preventing phagocytosis. In contrast with previous studies $(37,38)$, we found decreased levels in gliomas compared to normal brain. We consider that this down-expression can represent an attempt to maintain homeostasis. Recent studies have associated CD47 with the tumor-associated macrophages (TAMs) in the GBM microenvironment. Zhang et al (39) have also proven that anti-CD47 treatment leads to enhanced tumor cell phagocytosis by both M1 and M2 macrophage subtypes with a higher phagocytosis rate by M1 macrophages. A combination of anti-CD47 treatment and temozolamide has also been reported (40).

TNFRSF14 was found to be elevated in aggressive gliomas and its expression seemed to be associated with amplification of EGFR and loss of PTEN (41). TNFRSF14 plays an important role in the recruitment and activation of immune system in the tumor microenvironment. We showed that TNFRSF14 seems to have a significant impact on both the overall survival and the disease-free time. Interestingly, in metastatic melanoma, TNFRSF14 shows a similar behavior (42), further reinforcing our observations and suggesting that similar mechanisms can be shared also in glioma and that a combinatory blocking strategy can improve patients outcome.

Finally, we performed a deconvolution analysis showing that higher levels of CD276, TNFRSF14 and NECTIN2 are associated with a significant lower proportion of infiltrating plasma cells, while higher levels of VTCN1 were associated to higher proportions of infiltrating plasma cells, Th1, aDCs and cDCs. Higher levels of TNFRSF14 were associated with a major infiltration of iDCs, aDCs, pDCs and macrophages, but lower levels of Th1 cells and cDCs. Higher expression of NECTIN2, associated with shorter survival, is associated with reduced proportions of Th1, Th2 and CD8 T cells. Together these findings suggest that the main immune cell types that 
help to reduce the tumor mass and improve the survival are Th1 and cDCs, and that their expression is strictly dependent on these immune checkpoints. In agreement with our hypothesis, previous studies have shown that in gliomas, there is a prevalent Th2 response and that switching from Th2 to Th1 can help to block glioma growth (43). Additionally, recent studies have proven that combinational therapy that blocks more immune checkpoints is a possibility to create a more vigorous Th1 antitumor response $(44,45)$ and its association with better outcome (46). Future preclinical and clinical studies are necessary to ascertain whether, in addition to the prognostic value we have highlighted, the dysregulated expression of the inhibitory immune checkpoint presently studied may translate into clinical applications, as novel immunotherapeutic approaches for the treatment of gliomas and possibly other types of cancers.

Collectively, in this study, we evaluated the expression of several inhibitory immune checkpoints that can play a role in glioma progression. Among the investigated immune checkpoints, TNFRSF14 and NECTIN2 were identified as the most promising targets in GBM. In particular, TNFRSF14 expression is associated with worse overall survival and disease-free survival, correlating with a lower Th1 response and suggesting that it could become an interesting biomarker or therapeutic target.

\section{Acknowledgements}

Not applicable.

\section{Funding}

This study was supported by current research funds 2020 of IRCCS ‘Centro Neurolesi Bonino-Pulejo’, Messina, Italy.

\section{Availability of data and materials}

All the data in this study are available for download from TCGA (The Cancer Genome Atlas) databank.

\section{Authors' contributions}

Conceptualization: FN and PF; data curation: SDL, RB, KM and PF; formal analysis: MP and KM; funding acquisition: $\mathrm{AB}, \mathrm{PB}$ and $\mathrm{FN}$; investigation: $\mathrm{RC}$; project administration: $\mathrm{PB}$; supervision: FN; visualization: MSB; writing-original draft: SDL, RC, MSB, MP and RB; writing-review and editing: AB, $\mathrm{KM}, \mathrm{PB}, \mathrm{FN}$ and PF.

\section{Ethics approval and consent to participate}

Not applicable.

\section{Patient consent for publication}

Not applicable.

\section{Competing interests}

The authors declare that they have no competing interests.

\section{References}

1. Louis DN, Ohgaki H, Wiestler OD, Cavenee WK, Burger PC, Jouvet A, Scheithauer BW and Kleihues P: The 2007 WHO classification of tumours of the central nervous system. Acta Neuropathol 114: 97-109, 2007.

2. Bacher M, Schrader J, Thompson N, Kuschela K, Gemsa D, Waeber $G$ and Schlegel J: Up-regulation of macrophage migration inhibitory factor gene and protein expression in glial tumor cells during hypoxic and hypoglycemic stress indicates a critical role for angiogenesis in glioblastoma multiforme. Am J Pathol 162: 11-17, 2003.

3. Fritz L, Dirven L, Reijneveld JC, Koekkoek JA, Stiggelbout AM, Pasman HR and Taphoorn MJ: Advance care planning in glioblastoma patients. Cancers (Basel) 8: 102, 2016.

4. Huang B, Zhang H, Gu L, Ye B, Jian Z, Stary C and Xiong X: Advances in immunotherapy for glioblastoma multiforme. J Immunol Res 2017: 3597613, 2017.

5. Reardon DA, Wen PY, Wucherpfennig KW and Sampson JH: Immunomodulation for glioblastoma. Curr Opin Neurol 30 361-369, 2017.

6. Srinivasan VM, Ferguson SD, Lee S, Weathers S-P, Kerrigan BCP and Heimberger AB: Tumor vaccines for malignant gliomas. Neurotherapeutics 14: 345-357, 2017.

7. Sanders S and Debinski W: Challenges to successful implementation of the immune checkpoint inhibitors for treatment of glioblastoma. Int J Mol Sci 21: 21, 2020.

8. Aran D, Hu Z and Butte AJ: xCell: Digitally portraying the tissue cellular heterogeneity landscape. Genome Biol 18: 220, 2017.

9. Larkin J, Chiarion-Sileni V, González R, Grob J, Cowey C and Lao C: Combined nivolumab and ipilimumab or monotherapy in previously untreated melanoma. N Engl J Med 373: 23-34, 2015.

10. Ritprajak P and Azuma M: Intrinsic and extrinsic control of expression of the immunoregulatory molecule PD-L1 in epithelial cells and squamous cell carcinoma. Oral Oncol 51: 221-228, 2014.

11. Massari F, Santoni M, Ciccarese C, Santini D, Alfieri S, Martignoni G, Brunelli M, Piva F, Berardi R, Montironi R, et al: PD-1 blockade therapy in renal cell carcinoma: Current studies and future promises. Cancer Treat Rev 41: 114-121, 2015.

12. Tan AC, Heimberger AB and Khasraw M: Immune checkpoint inhibitors in gliomas. Curr Oncol Rep 19: 23, 2017.

13. Desai K, Hubben A and Ahluwalia M: The role of checkpoint inhibitors in glioblastoma. Target Oncol 14: 375-394, 2019.

14. Caccese $\mathrm{M}$, Indraccolo $\mathrm{S}$, Zagonel $\mathrm{V}$ and Lombardi $\mathrm{G}$ : PD-1/PD-L1 immune-checkpoint inhibitors in glioblastoma: A concise review. Crit Rev Oncol Hematol 135: 128-134, 2019.

15. Gustafsson M, Edström M, Gawel D, Nestor CE, Wang H, Zhang H, Barrenäs F, Tojo J, Kockum I, Olsson T, et al: Integrated genomic and prospective clinical studies show the importance of modular pleiotropy for disease susceptibility, diagnosis and treatment. Genome Med 6: 17, 2014.

16. Fagone P, Mazzon E, Cavalli E, Bramanti A, Petralia MC, Mangano K, Al-Abed Y, Bramati P and Nicoletti F: Contribution of the macrophage migration inhibitory factor superfamily of cytokines in the pathogenesis of preclinical and human multiple sclerosis: In silico and in vivo evidences. J Neuroimmunol 322 : 46-56, 2018.

17. Mangano K, Cavalli E, Mammana S, Basile MS, Caltabiano R, Pesce A, Puleo S, Atanasov AG, Magro G, Nicoletti F, et al: Involvement of the $\mathrm{Nrf} 2 / \mathrm{HO}-1 / \mathrm{CO}$ axis and therapeutic intervention with the CO-releasing molecule CORM-A1, in a murine model of autoimmune hepatitis. J Cell Physiol 233: 4156-4165, 2018.

18. Mammana S, Bramanti P, Mazzon E, Cavalli E, Basile MS, Fagone P, Petralia MC, McCubrey JA, Nicoletti F and Mangano K: Preclinical evaluation of the PI3K/Akt/mTOR pathway in animal models of multiple sclerosis. Oncotarget 9: 8263-8277, 2018.

19. Fagone P, Muthumani K, Mangano K, Magro G, Meroni PL, Kim JJ, Sardesai NY, Weiner DB and Nicoletti F: VGX-1027 modulates genes involved in lipopolysaccharide-induced Toll-like receptor 4 activation and in a murine model of systemic lupus ery thematosus. Immunology 142: 594-602, 2014.

20. Nicoletti F, Mazzon E, Fagone P, Mangano K, Mammana S, Cavalli E, Basile MS, Bramanti P, Scalabrino G, Lange A, et al: Prevention of clinical and histological signs of MOG-induced experimental allergic encephalomyelitis by prolonged treatment with recombinant human EGF. J Neuroimmunol 332: 224-232, 2019. 
21. Fagone P, Mazzon E, Mammana S, Di Marco R, Spinasanta F, Basile MS, Petralia MC, Bramanti P, Nicoletti F and Mangano K: Identification of $\mathrm{CD}^{+} \mathrm{T}$ cell biomarkers for predicting the response of patients with relapsing-remitting multiple sclerosis to natalizumab treatment. Mol Med Rep 20: 678-684, 2019.

22. Fagone P, Mangano K, Coco M, Perciavalle V, Garotta G, Romao CC and Nicoletti F: Therapeutic potential of carbon monoxide in multiple sclerosis. Clin Exp Immunol 167: 179-187, 2012.

23. Patti F, Cataldi ML, Nicoletti F, Reggio E, Nicoletti A and Reggio A: Combination of cyclophosphamide and interferon- $\beta$ halts progression in patients with rapidly transitional multiple sclerosis. J Neurol Neurosurg Psychiatry 71: 404-407, 2001.

24. Presti M, Mazzon E, Basile MS, Petralia MC, Bramanti A Colletti G, Bramanti P, Nicoletti F and Fagone P: Overexpression of macrophage migration inhibitory factor and functionally-related genes, D-DT, CD74, CD 44, CXCR2 and CXCR4, in glioblastoma. Oncol Lett 16: 2881-2886, 2018

25. Fagone P, Caltabiano R, Russo A, Lupo G, Anfuso CD, Basile MS, Longo A, Nicoletti F, De Pasquale R, Libra M, et al: Identification of novel chemotherapeutic strategies for metastatic uveal melanoma. Sci Rep 7: 44564, 2017.

26. Basile MS, Mazzon E, Russo A, Mammana S, Longo A, Bonfiglio V, Fallico M, Caltabiano R, Fagone P, Nicoletti F, et al: Differential modulation and prognostic values of immune-escape genes in uveal melanoma. PLoS One 14: e0210276, 2019.

27. Mangano K, Mazzon E, Basile MS, Di Marco R, Bramanti P, Mammana S, Petralia MC, Fagone P and Nicoletti F: Pathogenic role for macrophage migration inhibitory factor in glioblastoma and its targeting with specific inhibitors as novel tailored therapeutic approach. Oncotarget 9: 17951-17970, 2018.

28. Nicoletti F, Fagone P, Meroni P, McCubrey J and Bendtzen K: mTOR as a multifunctional therapeutic target in HIV infection. Drug Discov Today 16: 715-721, 2011.

29. Rothweiler F, Michaelis M, Brauer P, Otte J, Weber K, Fehse B, Doerr HW, Wiese M, Kreuter J, Al-Abed Y, et al: Anticancer effects of the nitric oxide-modified saquinavir derivative saquinavir-NO against multidrug-resistant cancer cells. Neoplasia 12: 1023-1030, 2010

30. Yao Y, Ye H, Qi Z, Mo L, Yue Q, Baral A, Hoon DSB, Vera JC, Heiss JD, Chen CC, et al: B7-H4(B7x)-mediated cross-talk between glioma-initiating cells and macrophages via the IL6/JAK/STAT3 pathway lead to poor prognosis in glioma patients. Clin Cancer Res 22: 2778-2790, 2016.

31. Yao Y, Luo F, Tang C, Chen D, Qin Z, Hua W, Xu M, Zhong P, Yu S, Chen D, et al: Molecular subgroups and B7-H4 expression levels predict responses to dendritic cell vaccines in glioblastoma: An exploratory randomized phase II clinical trial Cancer Immunol Immunother 67: 1777-1788, 2018.

32. Moertel CL, Xia J, LaRue R, Waldron NN, Andersen BM, Prins RM, Okada H, Donson AM, Foreman NK, Hunt MA, et al: CD200 in CNS tumor-induced immunosuppression: The role for CD200 pathway blockade in targeted immunotherapy. J Immunother Cancer 2: 46, 2014.

33. Wang CY,Hsieh YT, Fang KM, Yang CS and Tzeng SF: Reduction of CD200 expression in glioma cells enhances microglia activation and tumor growth. J Neurosci Res 94: 1460-1471, 2016.

34. Olin MR, Ampudia-Mesias E, Pennell CA, Sarver A, Chen CC, Moertel CL, Hunt MA and Pluhar GE: Treatment combining CD200 immune checkpoint inhibitor and tumor-lysate vaccination after surgery for pet dogs with high-grade glioma. Cancers (Basel) 11: 137, 2019.
35. Sloan KE, Stewart JK, Treloar AF, Matthews RT and Jay DG: CD155/PVR enhances glioma cell dispersal by regulating adhesion signaling and focal adhesion dynamics. Cancer Res 65 : 10930-10937, 2005.

36. Hung AL, Maxwell R, Theodros D, Belcaid Z, Mathios D, Luksik AS, Kim E, Wu A, Xia Y, Garzon-Muvdi T, et al: TIGIT and PD-1 dual checkpoint blockade enhances antitumor immunity and survival in GBM. OncoImmunology 7: e1466769, 2018.

37. Li F, Lv B, Liu Y, Hua T, Han J, Sun C, Xu L, Zhang Z, Feng Z, Cai Y, et al: Blocking the CD47-SIRP $\alpha$ axis by delivery of anti-CD47 antibody induces antitumor effects in glioma and glioma stem cells. OncoImmunology 7: e1391973, 2018.

38. Liu X, Wu X, Wang Y, Li Y, Chen X, Yang W and Jiang L: CD47 promotes human glioblastoma invasion through activation of the PI3K/Akt pathway. Oncol Res 27: 415-422, 2019.

39. Zhang M, Hutter G, Kahn SA, Azad TD, Gholamin S, Xu CY, Liu J, Achrol AS, Richard C, Sommerkamp P, et al: Anti-CD47 treatment stimulates phagocytosis of glioblastoma by M1 and M2 polarized macrophages and promotes M1 polarized macrophages in vivo. PLoS One 11: e0153550, 2016.

40. von Roemeling CA, Wang Y, Qie Y, Yuan H, Zhao H, Liu X, Yang Z, Yang M, Deng W, Bruno KA, et al: Therapeutic modulation of phagocytosis in glioblastoma can activate both innate and adaptive antitumour immunity. Nat Commun 11: 1508, 2020.

41. Han MZ, Wang S, Zhao WB, Ni SL, Yang N, Kong Y, Huang B, Chen AJ, Li XG, Wang J, et al: Immune checkpoint molecule herpes virus entry mediator is overexpressed and associated with poor prognosis in human glioblastoma. EBioMedicine 43 : 159-170, 2019.

42. Malissen N, Macagno N, Granjeaud S, Granier C, Moutardier V, Gaudy-Marqueste C, Habel N, Mandavit M, Guillot B, Pasero C, et al: HVEM has a broader expression than PD-L1 and constitutes a negative prognostic marker and potential treatment target for melanoma. OncoImmunology 8: e1665976, 2019.

43. Li G, Hu YS, Li XG, Zhang QL, Wang DH and Gong SF Expression and switching of TH1/TH2 type cytokines gene in human gliomas. Chin Med Sci J 20: 268-272, 2005.

44. Jahan N, Talat H, Alonso A, Saha D and Curry WT: Triple combination immunotherapy with GVAX, anti-PD-1 monoclonal antibody, and agonist anti-OX40 monoclonal antibody is highly effective against murine intracranial glioma. OncoImmunology 8 : e1577108, 2019.

45. Jahan N, Talat H and Curry WT: Agonist OX40 immunotherapy improves survival in glioma-bearing mice and is complementary with vaccination with irradiated GM-CSF-expressing tumor cells. Neuro-oncol 20: 44-54, 2018

46. Taube JM, Galon J, Sholl LM, Rodig SJ, Cottrell TR, Giraldo NA, Baras AS, Patel SS, Anders RA, Rimm DL, et al: Implications of the tumor immune microenvironment for staging and therapeutics. Mod Pathol 31: 214-234, 2018.

This work is licensed under a Creative Common Attribution-NonCommercial-NoDerivatives 4.0 International (CC BY-NC-ND 4.0) License. 